\title{
Clinical Decision Support System based on Fuzzy Cognitive Maps
}

Nassim Douali $^{1 *}$, Elpiniki I Papageorgiou ${ }^{2}$, Jos De Roo ${ }^{3}$, Hans Cools ${ }^{3}$ and Marie-Christine Jaulent

${ }^{1}$ INSERM UMR_S 872, Eq 20, Medicine Faculty, Pierre and Marie Curie University, Paris 6, France

${ }^{2}$ Department of Informatics and Computer Technology, Technological Educational Institute of Lamia, $3^{\text {rd }}$ Old National road Lamia-Athens, 35100 Lamia, Greece

${ }^{3}$ Agfa Health Care, Agfa Health Care NV, Moutstraat 100, 9000 Gent, Belgium

\begin{abstract}
Decision making in the field of medical diagnosis involves a degree of uncertainty and a need to take into account the patient's clinical parameters, the context of illness and the medical knowledge of the physician, to determine and confirm the diagnosis. In this study, we investigated and evaluated a model framework, for diagnostic decisions, based on a cognitive process and a Semantic Web approach. Fuzzy cognitive maps (FCM) are a cognitive process applying the main features of fuzzy logic and neural processors to situations involving imprecision and uncertain descriptions, in a similar way to intuitive human reasoning. We explored the use of this method for modeling clinical practice guidelines, using Semantic Web tools to implement these guidelines and for the formalization process. Twenty-five clinical and 13 diagnosis concepts were identified, to represent the problem of urinary tract infection diagnosis.
\end{abstract}

Keywords: Fuzzy cognitive maps; Decision making; Notation 3; Artificial intelligence; Decision support systems; Knowledge representation; Semantic web

\section{Introduction}

Diagnostic decision-making is not a simple task in medicine. The proposition and confirmation of a diagnosis must take into account the patient's clinical parameters, the clinical context and the physician's medical knowledge. Physicians use more than one million pieces of information in the care of their patients [1] and almost one third of their time is spent recording and compiling information [2]. Unsurprisingly, physicians may be unable to recall every item of relevant knowledge and to relate all these items to the care process [3]. Clinical decision support systems (CDSS) have been developed to improve patient safety and care processes $[4,5]$. Several studies and reviews have shown these tools to be effective [6-9] in the areas of diagnosis and treatment.

A decade ago, the United States consensus report "Crossing the Quality Chasm: A New Health System for the $21^{\text {st }}$ Century" highlighted the importance of using CDSS to reduce the frequency of medical errors, to ensure that best practice is followed and to reduce care costs [10]. In a recent review, Peleg and co-workers [3] suggested that CDSS should be a part of a knowledge-management toolkit that a healthcare organization can employ to deliver the "right knowledge to the right people in the right form at the right time" [11]. The development of CDSS involves two knowledge-management tasks: consideration of possibilities for the integration of the CDSS into the care system workflow and knowledge management for correct decision-making. Many problems arise in the content of knowledge bases and also of their quality. Clinical practice guidelines (CPG) are standardized documents developed to improve the quality of medical care. The computerization of clinical guidelines has attracted much interest in recent years, as it would not only facilitate their dissemination but also improve the knowledge-based process through which they are produced. There are several approaches to the computerization of clinical practice guidelines, which remains a very complex task [12]. We can classify them into two main categories [13]. The documentary approach: this approach is to use the document as a medium of representation; it is based on a markup text with markup languages like XML (Hypertext Guideline Markup Language (HGML) and Guideline Elements Model (GEM)). The second approach consists of extracting knowledge of CPG (Eon, GLIF, Asbru, Proforma, Prodigy and Arden Syntax MLM) [13].
CDSS models must take into account issues relating to patient data quality, such as incompleteness, poor structuring and lack of reliability and problems associated with inaccuracies and uncertainties in CPG. Many clinical diagnosis tasks involve reasoning in conditions of uncertainty [14], and there are many approaches for the uncertainty representation of knowledge in CPG, such as Bayesian networks (BN) [15] or fuzzy cognitive maps (FCM) [16]. FCM describe domains with nodes, also known as concepts (variables, states, inputs: facts, outputs: decision) and fuzzy relationships (the influence of concepts) between them. The fuzzy approach provides degrees of causality, represented as links between concepts (variables, states, inputs, outputs). This structure establishes the forward and backward propagation of causality, allowing the knowledge base to evolve through the addition, modification or removal of concepts and the links between them.

Urinary tract infection (UTI) is the most common urological disorder [17] and constitutes a serious health problem affecting millions of people each year [18]. UTI treatment costs billions of dollars annually [19]. UTI is the second most common type of infection [18] and the populations at particularly high risk of UTI include young children, pregnant women and the elderly, together with those with spinal cord injuries, indwelling catheters, diabetes, multiple sclerosis, human immunodeficiency virus and underlying urological abnormalities [20]. A study carried out in the United States and Canada showed that about a half of all women will have a UTI at some time in their lives, mostly caused by Escherichia coli, and a quarter of women will have recurrent infections [21]. A number of studies have shown that the diagnosis and management of UTI is a common, complex problem requiring special attention [21,22]. Inappropriate diagnosis and the massive

${ }^{*}$ Corresponding author: Nassim Douali, INSERM UMR_S 872, Eq 20, Medicine Faculty, Pierre and Marie Curie University ,Paris 6, France, Tel: 33-650154547; E-mail: nassim.douali@crc.jussieu.fr

Received October 21, 2014; Accepted March 02, 2015; Published March 05, 2015

Citation: Douali N, Papageorgiou El, Roo JD, Cools H, Jaulent MC (2015) Clinical Decision Support System based on Fuzzy Cognitive Maps. J Comput Sci Syst Biol 8: 112-120. doi:10.4172/jcsb. 1000177

Copyright: (C) 2015 Douali N, et al. This is an open-access article distributed under the terms of the Creative Commons Attribution License, which permits unrestricted use, distribution, and reproduction in any medium, provided the original author and source are credited. 
use of antibiotics are considered to be the key factors governing the development of bacterial resistance to antimicrobial drugs [20].

We present and discuss the results obtained for the application of the resulting diagnostic model to a database of 70 patients presenting with urinary tract infections.

\section{Background}

\section{Clinical decision support systems}

CDSS make a significant contribution to medical knowledge management technologies, by supporting the clinical process and the use of knowledge, from diagnosis and investigation, through to treatment and long-term care. Their role and acceptance in daily clinical practice is increasing [23]. Recent studies [24] have shown that CDSS can improve physician performance and accuracy, but that the quality of each system may depend on the technical approach used to model medical information. Schurink et al. (2005) reviewed a number of computer-assisted decision support systems for the diagnosis and treatment of infectious diseases in intensive care units [25]. They concluded that CDSS are useful for decision-making in infectious disease management, but that more prospective evaluation of these systems is required.

The choice of an empirical antimicrobial treatment suitable for the patient requires careful consideration of the patient's treatment parameters (current treatment, drug allergies etc.) but, above all, it requires a good first diagnosis based principally on a clinical approach. Diagnosis is based on a notion of causality between the infectious disease and its clinical signs in physiological and pathological conditions.

We aimed to create a computerized system that dynamically guides physicians through the workflow and helps them to diagnose clinical forms of UTI. We tackled the problem of modeling medical knowledge from guidelines and handling imprecision and uncertainty in the assignment of UTI diagnosis, through the combined use of cognitive maps and Semantic Web approaches.

\section{Fuzzy cognitive maps (FCM)}

FCM were developed by R. Kosko [26] as an extension of cognitive maps, to represent the cognitive relationships between concepts. FCM represent knowledge in a symbolic manner, encoding the relationships between the elements of a mental landscape so that the impact of these elements can be assessed. FCM applies fuzzy logic to cognitive maps, making it possible to predict changes in the concepts represented in cognitive maps. The graphical illustration of FCM is a signed, directed graph with feedback, consisting of nodes and weighted interconnections. Nodes correspond to concepts: variables and states used to describe the behavior of the system.

Nodes are connected by weighted arrows representing cognitive relationships between nodes (Figure 1). Each concept is characterized by a range of value, generally $[0 \ldots 1]$ or $[-1 \ldots 1]$. The dynamics of the system is implied by the interaction of concepts through the cognitive strength of relationships among them. The cognitive relationships are represented linguistically with an associated fuzzy set. It allows having degrees of causality. This structure establishes the forward and backward propagation of causality, allows the knowledge base to increase when concepts and links between them are increased [27,28].

The construction of an FCM for the modeling of a medical decision making task is consisting of two parts: (a) the determination of concepts and (b) the determination of the strength of cognitive

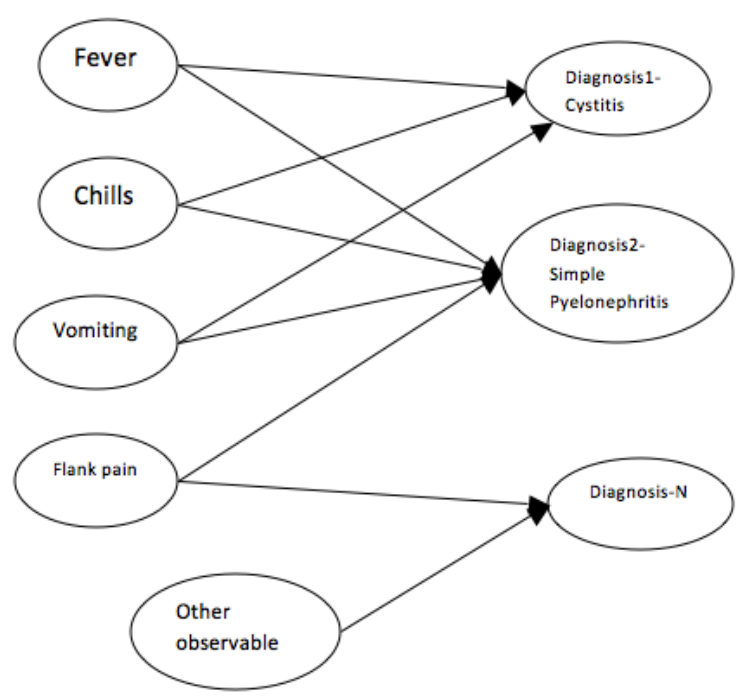

Figure 1: Representation of an FCM model.

relationships between concepts. Each concept is modeled as a variable $C_{i}, i=1,2, \ldots N$ that can take fuzzy or discrete values according to the problem data and is determined by experts' knowledge and medical guidelines. The strengths of cognitive relations (namely weights) between concepts are assigned by if-then rules, which are constructed by experts-physicians based on medical guidelines. There are three possible types of cognitive relationships between concepts, expressing the type of influence of one concept on others. The weight assigned to the interconnection between concept $\mathrm{C}_{i}$ and concept $\mathrm{C}_{\mathrm{j}}$, denoted by $\mathrm{W}_{i \mathrm{j}}$ (wrights), is positive $\left(\mathrm{W}_{\mathrm{ij}}>0\right)$ for positive causality or negative $\left(\mathrm{W}_{\mathrm{ij}}<0\right)$ for negative causality. Alternatively, it could be null $\left(\mathrm{W}_{\mathrm{ij}}=0\right)$, if there is no relationship between concept $\mathrm{C}_{i}$ and concept $\mathrm{C}_{i}$. Knowledge about the cognitiverelationships controlling the dynamic behavior of the system is stored in the structure of a map and in the interconnections summarizing the correlations between causes and effects.

The cognitive relationships between concepts were initially determined by experts following the construction process of FCMs, as linguistic variables [28]. The linguistic variables proposed in [27] and used for modeling FCMs are: $T$ (influence) $=\{$ negatively very strong, negatively strong, negatively medium, negatively weak, negatively very weak, zero, positively very weak, positively weak, positively medium, positively strong, positively very strong, positively very very strong\}. These twelve triangular membership functions have been previously described in $[28,29]$ showing their functionality. The fuzzy influences among concepts are transferred to numerical values of connections, called weights $\mathrm{W}_{\mathrm{ij}}$, which are estimated by defuzzification of the aggregated, linguistically expressed, concept connections.

In semantic web, the weights $\mathrm{W}_{\mathrm{ij}}$ are transferred to semantic weights, namely $\mathrm{W}_{\mathrm{ij}}$ semantic within the range $[0,1]$ that depicts the semantic value of numerical weight. Once the FCM is constructed, it can receive data from its input concepts, perform reasoning and infer medical decisions as values of its output concepts [29,30]. A generic diagnosis example is illustrated in Figure 1.

The value of a concept $\mathrm{D}$ is obtained by multiplying the value of each of its input concepts, $\mathrm{C}_{\mathrm{i}}$, by their respective weights, $\mathrm{W}_{\mathrm{ij}}$, giving values of $[-1 . .1]$. The values are then summed and a non linear function 
is used to limit the range of possible output values.

\section{Fuzzy Cognitive Map reasoning mechanism}

The FCM reasoning process follows a number of steps till the system's equilibrium point. These steps can be found in $[28,30]$ and we briefly present them here. At first step, the initial state of the concepts is given either from experts or from the existing medical database. During reasoning the FCM iteratively calculates its state until convergence. The state is represented by a state vector $C^{k}$, which consists of real node values $C_{i}^{(k)} \in[0,1], i=1,2, \ldots N$ at an iteration $k$. The value of each node is calculated by the following equation:

$$
C_{i}^{(k+1)}=f\left[C_{i}^{(k)}+\sum_{\substack{j \neq i \\ j=1}}^{N} C_{i}^{(k)} \cdot W_{j i}\right]
$$

where $f$ is a threshold (activation) function:

$$
f(x)=1 /\left(1+e^{-m(x)}\right)
$$

Where $m$ is a constant parameter [28]. The parameter $m$ determines how quickly the $f(x)$ approaches the limiting values of 0 and 1 . The transformation function is used to reduce unbounded weighted sum to a certain range, which hinders quantitative analysis, but allows for qualitative comparisons between concepts [30].

In order to remove the spurious influence of inactive concepts (concepts with zero values) on other concepts, and to avoid the conflicts emerge in cases where the initial values of concepts are 0.5 , as well as the missing data, a modified FCM reasoning formalism can be used. Based on this assumption, we reformulated eq. (1) as:

$$
C_{i}^{(k+1)}=f\left(\left(2 C_{i}^{(k)}-1\right)+\sum_{\substack{j \neq i \\ j=1}}^{N}\left(2 C_{j}^{(k)}-1\right) \cdot W_{j i}\right)
$$

This eq. (3) overcomes also the limitation present by the sigmoid threshold function. Thus, the insufficient knowledge and/or missing information for each node can be handled with less deviation from reality.

The simulation stops when a limit vector is reached, i.e., when $C_{i}^{k}-C_{i}^{k-1} \leq e$; where $e$ is a residual, whose value depends on the application type (and in most applications is equal to 0.001) [30]. The conclusions based on FCM should be viewed together with existing scientific knowledge [31,32]. Conclusions based on an analysis and/ or simulations of FCM can be counter-intuitive or against scientific results. If such are encountered, one must further study the assumptions depicted in FCMs, but also be open to insights gained from a systemic approach to problem analysis that FCM is.

\section{Materials and Methods}

\section{Materials}

In our project, resources are represented by the clinical data ${ }^{1}$, diagnostic guidelines (in the form of a decision tree) for urinary tract infections and domain ontology. We used 70 anonymous patients from several European hospitals. From this data set, 32 patients were female and 38 male with an average age $44 \pm 25$ (average \pm standard dev) twelve of 32 female patients were pregnant. For each patient, signs/symptoms/ observables/age/sex were taken into account by the system,we used the decision tree created manually in our project from the AFSSAPS and European Association of Urology CPG. We worked with the domain ontology of Infectious Diseases DCO (Debug IT Core Ontology) [31]. DCO serve as the core component of the interoperability platform for the Debug IT project ${ }^{2}$.

\section{Inference engine}

As part of our project, we aim to formalize the rules and have them executed by an inference engine. EulerSharp (known as Eye) [32] is an inference engine with backward-forward-backward chaining that allows "loop-checking" to ensure the processing of all data, to reason with data, queries and rules in N3. We chose to use EulerSharp on the basis of its historical performance ${ }^{3}$, frequent updates ${ }^{4}$, plugins and the possibility of integrating new features ${ }^{5}$ (built-in).

\section{Methods}

Our approach involves the various phases of cognitive map construction and the use of these maps with Semantic Web tools.

Figure 2 shows the steps involved in the formalization of guidelines in $\mathrm{N} 3$ and their connection with the final CDSS used directly by the physician. We present in detail the steps followed during the design of our system.

Structuring and enhancement of the decision tree: This is the first step in the proposed approach. We initially worked with the AFSSAPS (French Agency for the Safety of Health Products) $\mathrm{CPG}^{6}$, subsequently integrating a second CPG (European Urology Association). A medical expert will read and make the decision tree from the CPG.

Extraction of clinical concepts and diagnoses: We extracted clinical concepts and diagnostic concepts (all diagnoses) from our decision tree. In the decision tree, all attributes will be extracted as the concept. There are two types of concepts: diagnosis concepts (diseases.) and clinical concepts (clinical, biologicaland radiological). The disease is manifested by clinical signs but in our diagnostic approach, we deduce the dignosis from the clinical findings or signs (Figure 3).

Standardization of clinical concepts and diagnoses: We manually standardized concepts with the DebugIT ontology of Infectious Diseases (DCO).Each attribute is aligned with a concept of ontology DCO (Figure 4).

Writing diagnostic rules: The CPG is expressed as "If Premise Then Conclusion" rules. The premise takes into account several patient parameters and consists of several basic premises. The extraction of knowledge from CPG takes the form of fuzzy "If-Then" rules, which can be formalized as follows:

If the value of the concept $\mathrm{Ci}$ is "a state $\mathrm{X}$ "/increases/decreases then the value of $\mathrm{Y}$ is $\mathrm{D}_{i}$, with a degree of influence (weight) $\mathrm{W}_{\mathrm{ij}}$. Every relationship between a concept and a clinical diagnostic concept is defined as a membership function determining the degree of causality between two concepts (see below). For example, "If the urine dipstick

1Data bases from several European hospitals (France, Germany, Belgium).

${ }^{2}$ Debuglt: The DebuglT project is a large -scale integrating project funded within the $7^{\text {th }}$ EU Framework Programme (FP7)

${ }^{3} \mathrm{http}: / /$ eulersharp.sourceforge.net/2003/03swap/dtb-2010.txt.

${ }^{4} \mathrm{http}: / /$ eulersharp.sourceforge.net/DONE.

${ }^{5} \mathrm{http}: / /$ eulersharp.sourceforge.net/2003/03swap/euler-builtins.html.

${ }^{6} \mathrm{http}: / /$ www.afssaps.fr/Infos-de-securite/Recommandations-de-bonnepratique/Diagnostic-et-antibiotherapie-des-infections-urinaires-bacteriennescommunautaires-de-l-adulte-recommandations-de-bonne-pratique. 
Citation: Douali N, Papageorgiou El, Roo JD, Cools H, Jaulent MC (2015) Clinical Decision Support System based on Fuzzy Cognitive Maps. J Comput Sci Syst Biol 8: 112-120. doi:10.4172/jcsb.1000177
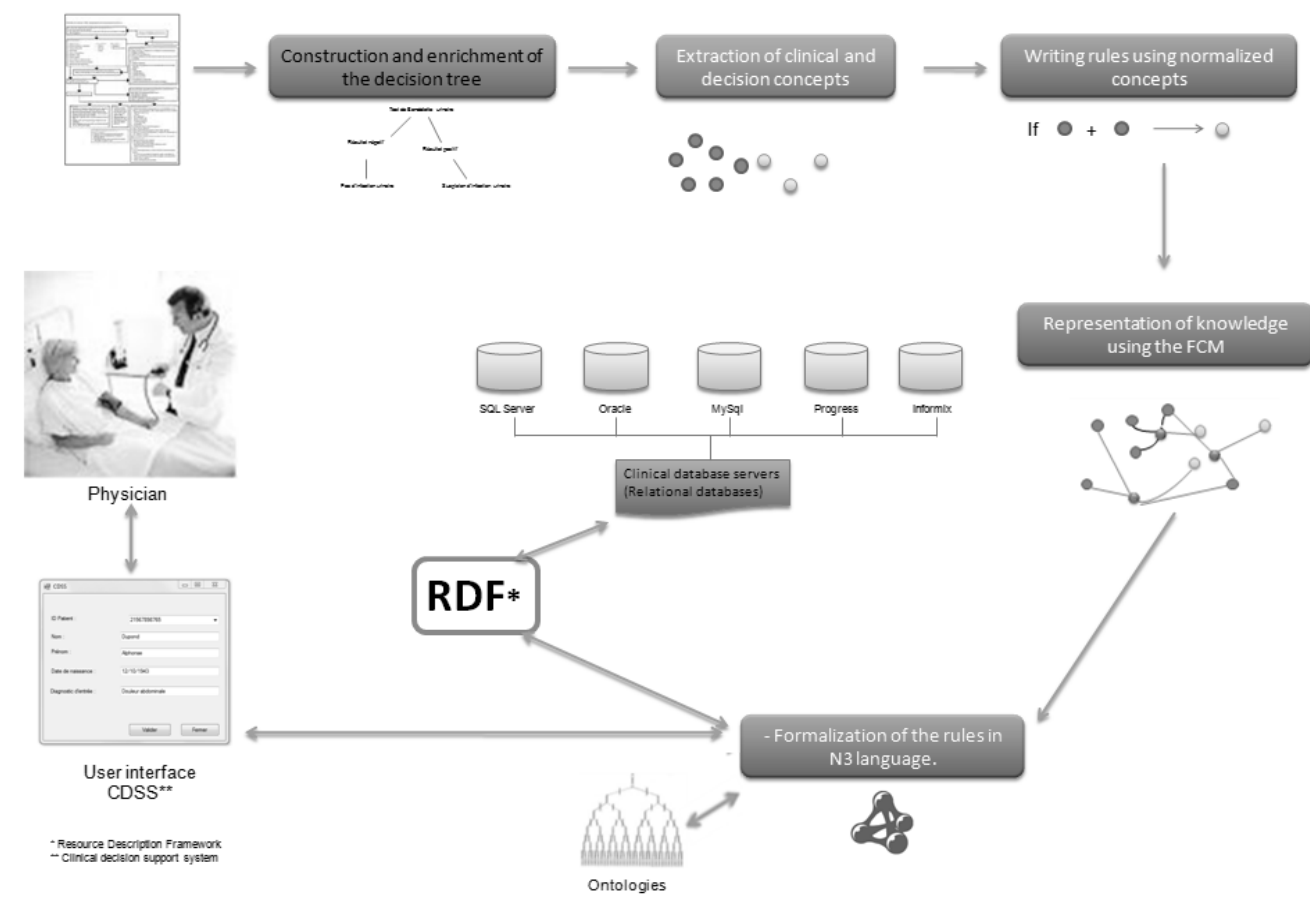

Figure 2: Diagram showing the approach used for CPG implementation.

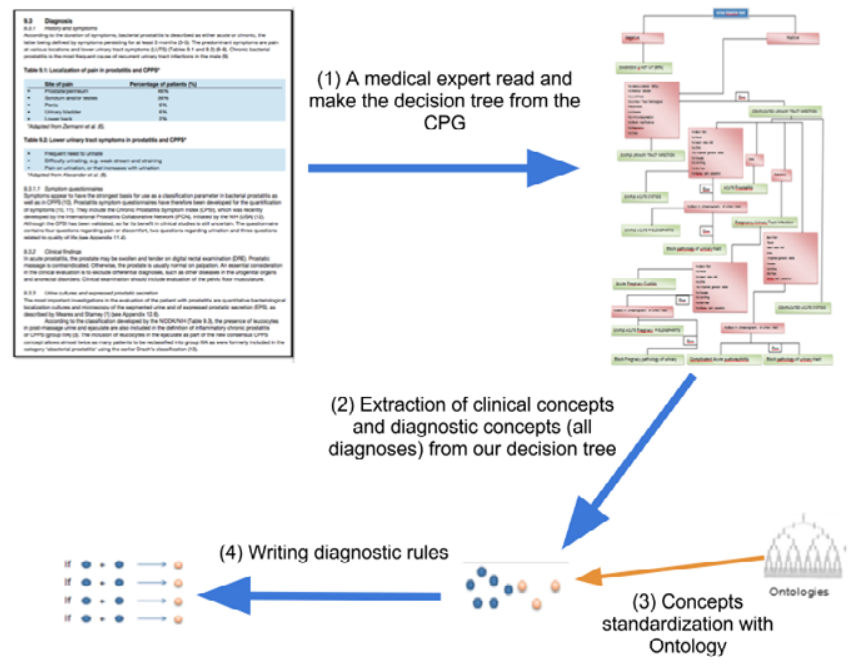

Figure 3: Diagram showing the approach used for CPG implementation.

test is negative then the diagnosis of UTI is wrong with a degree of confidence of 0.9 ". Our approach makes use of this knowledge to build cognitive maps in which these rules represent the relationships between concepts and a fuzzy value is attributed to the weight of each relationship.

Construction of an FCM model for UTI diagnosis: We first developed FCM for UTI diagnosis based on the CPG. A number of medical guidelines relating to the diagnosis of UTI were formalized into FCM knowledge models, according to the approach presented in Figure 4. For the construction of our FCM model for medical decision support, it was necessary to identify the main concepts and to determine the strengths of connections (cognitiveinfluences) between

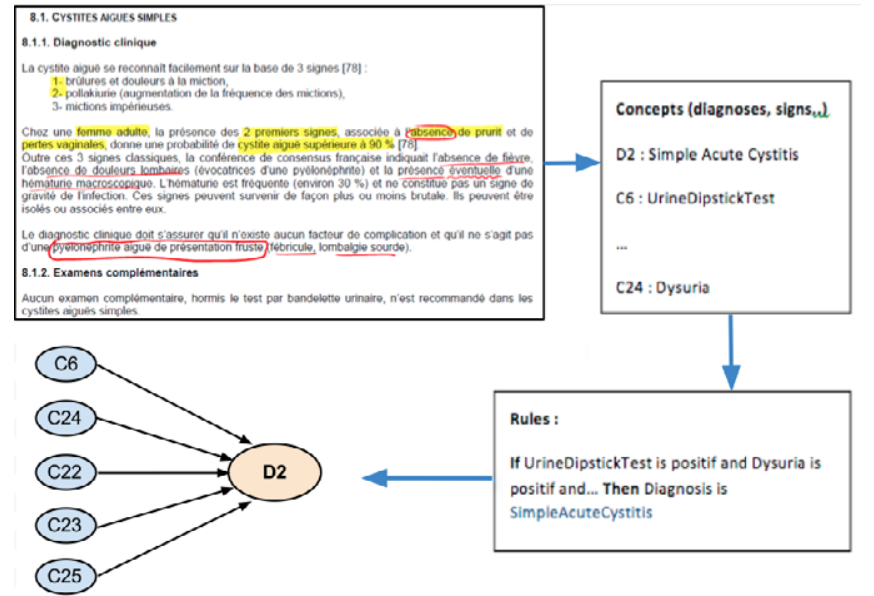

Figure 4: Diagram describing the elementary FCMconstruction.

these concepts. It was also necessary to extract a number of IF-THEN rules for the improvement of differential diagnosis. The concepts and their relationships were extracted manually, initially from CPG, using the previously described decision tree approach. Each concept and clinical diagnosis contributes to a node represented in FCM.

By this way, all the concepts were defined. The relationships between these concepts and the clinical diagnosis concepts described in the natural language of the CPG were translated into formal language by defining the fuzzy weight of each concept [30]. These linguistic weights were initially used to describe the influence of a clinical sign (concept) on a clinical diagnosis. These weights were then converted into numerical weights in the range $[-1,1]$, by the centroid defuzzification method of fuzzy logic. 


\section{The clinical and diagnostic concepts}

For the UTI diagnosis problem considered here, we defined 30 clinical concepts corresponding to signs, symptoms, observations and examinations. Thirteen diagnostic concepts corresponding to possible diagnoses of UTIs were described from CPGs.

FCM model weights: In FCM models, weights (initially described by fuzzy membership functions) can take their values within the range $[-1,1]$, but in $\mathrm{N} 3$ these values must be translated to the range 0 to 1 , with conservation of their meaning and ensuring that the same results are obtained. Each of the FCM weights indicates a degree of confidence, from 0 to 1 (Table 1$)$.

Elementary FCM for each possible UTI diagnosis: For each possible UTI diagnosis, we constructed an elementary FCM (subFCM) describing the diagnosis according to the initial conditions. We therefore constructed 13 elementary FCM.

We represented the concepts, with their causality, in 13 elementary interconnected FCM. The concepts " $\mathrm{C}_{i}$ " are the clinical concepts and $\mathrm{D}_{j}$ is the diagnostic concept described in Figure 4.

FCM implementation in N3: We implemented our algorithm for reasoning in N3, using plugins of the inference engine EulerSharp developed in Prolog ${ }^{7}$.

Medical models are written in the form of rules. This simplifies the modeling technique to the expression of relationships in terms of True or False structures and the attachment of degrees of confidence in the relationship. Furthermore, rules expressing medical knowledge make use of built-in elements of the Eye [32]. For FCM modeling, the following Eye predicates are used with the Euler built-in fl:pi.

- fl:mu is an Eye predicate for expressing fuzzy set membership, e.g. (:x :C) fl:mu 0.8 indicates that $: \mathrm{x}$ is a $: \mathrm{C}$ with a degree of confidence in fuzzy set membership of 0.8 .

For example:

\section{(:patient001:Symptom01)fl:mu 1}

This indicates that the patient suffers from Symptom01, and the activation value of FCM concept "Symptom01" is 1 . The expression fl:mu 1 "bridges" the relationship between the Symptom01 and the patient001 by creating fuzzy set membership.

- $\quad \mathrm{fl}:$ sigma is an Eye predicate for expressing fuzzy subsethood

\begin{tabular}{|c|c|c|c|}
\hline $\begin{array}{l}\text { Fuzzy membership functions } \\
\text { (triangular) }\end{array}$ & $\begin{array}{l}\text { Fuzzy } \\
\text { regions }\end{array}$ & $\begin{array}{l}\text { Deffuzzified } \\
\text { value (weight) }\end{array}$ & $\begin{array}{l}\text { Semantic } \\
\text { weights }\end{array}$ \\
\hline $\begin{array}{l}\text { Negative very very strong } \\
\text { influence }\end{array}$ & {$[-1,-0.9)$} & -1 & 0 \\
\hline Negative very strong influence & $(-0.9-0.7)$ & -0.8 & 0.05 \\
\hline Negative strong influence & $(-0.8-0.6)$ & -0.7 & 0.1 \\
\hline Negative medium influence & $\left(\begin{array}{ll}-0.7 & -0.4\end{array}\right)$ & -0.55 & 0.15 \\
\hline Negative weak influence & $\left(\begin{array}{ll}-0.4 & -0.2\end{array}\right)$ & -0.3 & 0.2 \\
\hline Negative very weak influence & $\left(\begin{array}{ll}-0.2 & 0\end{array}\right)$ & -0.1 & 0.3 \\
\hline Positive very weak influence & $\left(\begin{array}{ll}0 & 0.2\end{array}\right)$ & 0.1 & 0.4 \\
\hline Positive weak influence & $\left(\begin{array}{ll}0.2 & 0.4\end{array}\right)$ & 0.3 & 0.55 \\
\hline Positive medium influence & $(0.40 .7)$ & 0.55 & 0.65 \\
\hline Positive strong influence & $\left(\begin{array}{ll}0.6 & 0.8\end{array}\right)$ & 0.7 & 0.75 \\
\hline Positive very strong influence & $\left(\begin{array}{ll}0.7 & 0.9\end{array}\right)$ & 0.8 & 0.85 \\
\hline Positive very very strong influence & $(0.91)$ & 1 & 1 \\
\hline
\end{tabular}

Table 1: Determination of fuzzy weights for semantic languages.

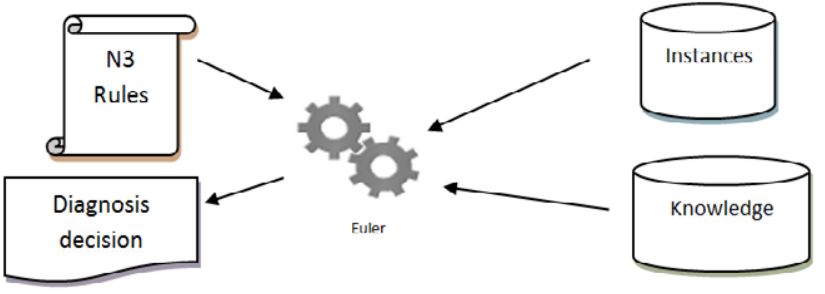

Figure 5: Representation of the mechanism for diagnosis decision-making .

e.g. (:C :D) fl:sigma 0.9 indicates that $: C$ is a rdfs:subClassOf $: D$ with a degree of confidence of 0.9 .

A concrete example for the FCM disease model would be:

(:Symptom01:D1-diagnosis) fl:sigma 0.3.

- This indicates that the observable "Symptom01" has a strength of impact on "D1-diagnosis" of 0.3 (the numerical value for fuzzy set membership). This value of 0.3 expresses the defuzzified value of weight (strength of the relationship) between these two concepts.

fl:pi is an Euler built-in rdf: Property for expressing the reasoning process of FCM. fl:pi is a built-in supplied via plug-in http://eulersharp. sourceforge.net/2006/02swap/fcm-plugin.yap.

The formalized medical knowledge in the context of FCM is represented with ontological concepts, using N3 and logic-rules format.

The FCM concepts are included in an ontology repository established for the needs of the project: DCO. DCO is continually updated to ensure that it contains all the relevant clinical concepts. We worked with the DebugIT project ontologists to enrich the DCO ontology for use with our rules (Figure 5).

Thus, based on this implementation approach, all the concepts, relationships and fuzzy rules for differential diagnosis are written in N3. The case of a patient is shown below as an example.

Case: Male, Old, Digital Rectal Examination (positive), Urine Dipstick Test (positive), Dysuria, Urinary Frequency, Residual Bladder.

With the proposed methodology, each cause-effect relationship between an observation and diagnosis is described by one or more fuzzy rules, thus producing the rule-based FCMs. From each fuzzy rule, an inference is generated, depicting the degree of confidence we have in the influence concerned. Moreover, based on the available CPG encoded as fuzzy rules, some of the relationships (weights) may change in value or degree of confidence before the final diagnosis is reached, taking into account cases and observable states that contribute to a different diagnosis. This is an important issue in FCM construction and diagnosis, as specific initial states of observables can be assessed for patients (Table 2).

In Appendix A, some examples of the FCM formalization of UTI diagnosis problems in N3 are given.

We investigated the use of this tool to provide diagnostic patterns for UTIs. The proposed approach, using FCMs, is able to establish decisions for the differential diagnosis of patients presenting UTI symptoms.

\section{Results}

The result of our work is summarized in the development of 


\begin{tabular}{|l|l|l|l|}
\hline \multicolumn{2}{|c|}{ Rule } & Impact of influence & Formalization in N3 \\
\hline Rule1 & $\begin{array}{l}\text { If the patient is Pregnant, then } \\
\text { Simple Urinary Tract Infection is an } \\
\text { inadequate diagnosis }\end{array}$ & $\begin{array}{l}\text { The strength of influence between "Simple Urinary } \\
\text { Tract Infection" and "Pregnant" is zero (no relationship } \\
\text { between concepts in the case of this patient) }\end{array}$ & (dco:Pregnant dco:SimpleUrinaryTractInfection) fl:sigma 0. \\
\hline Rule 2 & $\begin{array}{l}\text { If patient has Flank Pain THEN the } \\
\text { patient may have a Complicated } \\
\text { Urinary Tract Infection }\end{array}$ & $\begin{array}{l}\text { The strength of the relationship between Flank Pain and } \\
\text { Complicated Urinary Tract Infection is positive and very } \\
\text { very strong (numerical value=1) }\end{array}$ & $\begin{array}{l}\text { The strength of the relationship between Flank Pain and } \\
\text { Complicated Urinary Tract Infection is positive and very very } \\
\text { strong (numerical value=1) }\end{array}$ \\
\hline
\end{tabular}

Table 2: Example of Formalization of rules in N3.

a platform able to interact with heterogeneous data and formalize knowledge from CPG. The results presented here follow the steps in the methodology described above and the inference mechanism for FCM (Eulersharp/plugin). 92\% (65/70 patients: 37 males and 28 females) diagnosis proposed by the system was in fully agreement with the guidelines.

\section{Inference results}

We present an overview of the RDF file containing the results generated by the inference engine. For example, patient 013 is very likely to have a urinary tract infection but it is most likely to have prostatitis.

Patient 13 (symptoms): Male, Old, Urine Dipstick Test (positive), Dysuria, Urinary Frequency, Residual Bladder, Vesical Urethral Reflux. These symptoms are translated in $\mathrm{N} 3$ as follows:

(:patient0013 dco:FlankPain) fl:pi 0.

(:patient0013 dco:Fever) fl:pi 0.

(:patient0013 dco:Male) fl:pi 1.

(:patient0013 dco:UrineDipstickTest) fl:pi 1.

(:patient0013 dco:Chills) fl:pi 0.

(:patient0013 dco:Pregnant) fl:pi 0.

(:patient0013 dco:DisorderOfUrinaryTract) fl:pi 1.

(:patient0013 dco:Dysuria) fl:pi 1.

(:patient0013 dco:UrinaryFrequency) fl:pi 1.

(:patient0013 dco:ResidualBladder) fl:pi 1.

(:patient0013 dco:VesicalUretralReflux) fl:pi 1.

(:patient0013 dco:Urolithiasis) fl:pi 0.

(:patient0013 dco:DiabetesMellitus) fl:pi 0.

(:patient0013 dco:Immunosuppression) fl:pi 0.

(:patient0013 dco:Kidneyinsuffiency) fl:pi 0.

(:patient0013 dco:PathologicalState) fl:pi 0.

(:patient0013 dco:Old) fl:pi 1.

Using Euler Yap inference engine the following results are produced (Figure 6):

The numerical values from 0 to 1 indicate the rank of the suggested diagnosis. Diagnosis concept values with values close to 1 are the most likely to be correct, following the CPG list closely. A diagnostic concept value close to 0 indicates that the corresponding diagnosis is highly unlikely. In the results of the patient 013 displayed in Figure 6, we notice the presence of two diagnoses with a 0.99 degree of belief and the third one has 0.59 . We take the two diagnoses that the same
918 (:patient013 dco:SimpleUrinaryTractInfection) f1:pi 0.00248491163136268 . 919 (:patient013 dco: SimpleAcuteCystitis) f1:pi 0.0228824879380867 . 920 (:patient013 dco:Comp 1icatedUrinaryTractInfection) fl:pi 0.999815860305149. 221 (:patient013 dco: SimpleAcutePyelonephritis) $f 1$ : pi 0.156053000586995 , 22. (:patient013 dco:ComplicatedAcutePyelonephritis) $\mathrm{fl}: \mathrm{pi} \quad 0.249584057063839$. 223 (:patient013 dco:PregnancyUrinaryTractInfection) f1:pi 0.0280908538257439. 24 (:patient013 dco: ObstructiveUropathy) $f 1:$ pi 0.19680364389323 25 (:patient013 dco: Pregnancy0bstructiveUropathy) f1:pi 0.317608912015648. 926 (:patient013 dco: ComplicatedAcutePregnancyPyelonephritis) $11:$ pi 0.597472529811125. 927 (:patient013 dco: AcuteProstatis) $\quad[1:$ pi 0.999876574964054.

Figure 6: An overview of inference results (acute prostatitis).

disease because the concept "Acute Prostatis" is a son of the concept "Complicate urinary tract infection" (Acute prostate is a complicate urinary infection).

As this work concerns the modeling of diagnosis guidelines, the results obtained were compared with the recommendations provided by the relevant clinical suggestions.

\section{Discussion}

This study had three main objectives. The first was to establish reasoning algorithms for fuzzy cognitive maps. The second was to use $\mathrm{N} 3$ notation and logic to implement of our cognitive maps for CPG knowledge formalization and the integration of other knowledge resources (other CPG). Finally, we validated the model by applying our cognitive map reasoning to a patient database.

The proposed approach made it possible to model the medical knowledge contained in CPGs and to identify cognitive relationships through a process resembling human reasoning as closely as possible.

By using fuzzy cognitive maps, we were able to incorporate a second source of knowledge complementary to the AFSSAPS CPG. The possibility of incorporating other types of knowledge is advantage as it makes it possible to model simple knowledge in a medical field that is broad, complex and closely related to other areas.

We implemented the knowledge bases, rules and databases in the same environment (RDF, N3, Euler etc.) without compatibility constraints. This is one of the advantages of using Semantic Web tools. The success rate of the proposed model for UTI diagnosis was $92 \%$, demonstrating that this model is functional and likely to be useful in clinical practice in the future. The advantage of this approach is that it facilitates the sharing and reuse of knowledge from CPG and simplifies maintenance.

One advantage of the proposed FCM-based decision-making system over other approaches, such as the Bayesian belief networks, artificial neural networks, logistic regression or even other fuzzy logic-based approaches, is that it resembles human decision-making, with its capacity for approximate reasoning and handling incomplete information. We used only one source of knowledge appropriate to CPG. The field of community-acquired infections of the urinary tract in adults is not entirely covered by CPG, and we identified a large amount of missing information concerning the relationship between 
domain concepts. The rules implemented concerned only communityacquired urinary tract infections in adults. The integration of other CPG covering other infectious diseases domains would improve this system.

All fuzzy systems have a capability to translate the human operator knowhow into expertise rules expressed in a simple language makes it a very promising technique. Nevertheless, when the number of variables to be considered becomes too high, the rule base rapidly explodes, and problems linked to its implementation follow. The problem of the combinatorial explosion in the number of rules is present.

Moreover, the knowledge held in CPG is not sufficient for the diagnosis of and response to various specific and clinical situations. Thus, the knowledge contained in the guidelines is not sufficient in itself to produce a dynamic and efficient tool for decision support in real clinical practice. Future work should focus on the construction of more dynamic systems based on the proposed methodology, which could potentially integrate more sources of knowledge and data covering other infectious diseases.

\section{Conclusion}

We present here our results for the modeling of medical knowledge/ guidelines and the behavior of the system for decision support in UTI diagnosis based on the use of a new soft computing technology consisting of FCMs implemented in a Semantic Web approach. This work establishes a decision support tool based on FCM formalism for UTI diagnosis, by proposing the appropriate diagnosis for each individual case. 2 CPGs for the diagnosis of UTI were formalized into elementary FCM, to establish the FCM knowledge model. FCM methodology was then implemented in $\mathrm{N} 3$, which is an open and semantic language. The developed software tool determines diagnosis recommendations for UTIs. It receives relevant data concerning the state of the patient as input from the data store or a clinical information system (CIS). The simulations for test patients performed by implementation of the proposed FCMs provide the differential diagnoses for this pilot set of patients.

In this study we investigated the use of cognitive approaches for implementing CPG with Semantic Web tools. This work represents a first step towards the development of a methodology for the implementation of dynamic heterogeneous knowledge in medicine for decision support systems. In future studies [33-50], we plan to test our rules on larger data sets and to update them by integrating knowledge from experts and data mining.

\section{Acknowledgment}

The DebuglT project (http://www.debugit.eu/) is funded by the European Community's Seventh Framework Program for Research and Development, under grant agreement no. FP7-217139. The information in this document reflects solely the views of the authors and no guarantee or warranty is given that it is fit for any particular purpose. The European Commission, Directorate General for the Information Society and Media, Brussels, is not liable for any use made of the information contained in this article.

\section{Appendix A}

Some of our UTI diagnosis knowledge base is presented in N3. For example:

(:patient001:Dysuria)fl:mu 1

This means that the patient suffers from "Dysuria". Dysuria is a concept of the FCM disease model describing a patient condition "patient001" and the activation value of the FCM concept "Dysuria" is 1.

(:patient001:Strangury) fl:mu 0
This means that the patient does not suffer from "Strangury" (the symptomconcept of disease model is "Strangury") and the activation value of concept "Strangury" is 0 .

(:patient001:Pregnancy) fl:mu 1.

This means that the patient (female) is pregnant, and the activated value of concept "Pregnancy" is 1.

Another example, for the relationship between an observable and diagnosis concept:

(:C3-Dysuria:D1-UTI-diagnosis) fl:sigma 0.3.

This means that the observable "Dysuria" has an impact on "D1-diagnosis" with a confidence level of 0.3 (numerical value of fuzzy set membership). The value of 0.3 is the defuzzified value of fuzzy set membership defining the weight (strength of relationship) between these two concepts.

For example, the N3 code for the model of simple acute pyelonephritis is as follows:

\# Definition of the relationship between clinical concepts and simple acute pyelonephritis

(dco:FlankPain dco:SimpleAcutePyelonephritis) fl:sigma 1.

(dco:Fever dco:SimpleAcutePyelonephritis) fl:sigma 1.

(dco:Pregnant dco:SimpleAcutePyelonephritis) fl:sigma 0.

(dco:UrineDipstickTest dco:SimpleAcutePyelonephritis) fl:sigma 1.

(dco:PainfulKidneysPalpation dco:SimpleAcutePyelonephritis) fl:sigma 1.

(dco:Male dco:SimpleAcutePyelonephritis) fl:sigma 0.

(dco:Chills dco:SimpleAcutePyelonephritis) fl:sigma 1

(dco:Urolithiasis dco:SimpleAcutePyelonephritis) fl:sigma 0.

(dco:UrinaryFrequency dco:SimpleAcutePyelonephritis) fl:sigma 0.4 .

(dco:HeartPulseRate dco:SimpleAcutePyelonephritis) fl:sigma 0.8.

(dco:SuprapubicPain dco:SimpleAcutePyelonephritis) fl:sigma 0.5.

(dco:Dysuria dco:SimpleAcutePyelonephritis) fl:sigma 0.6.

(dco:SuprapubicPain dco:SimpleAcutePyelonephritis) fl:sigma 0.6.

$\{($ Pdco:FlankPain)fl:mu0. $\}=>\{($ dco:FlankPaindco:SimpleAcutePyelonephritis $)$ fl:sigma 0.

(dco:Feverdco:SimpleAcutePyelonephritis) fl:sigma 0.

(dco:UrineDipstickTest dco:SimpleAcutePyelonephritis) fl:sigma 0.

(dco:PainfulKidneysPalpation dco:SimpleAcutePyelonephritis) fl:sigma 0.

(dco:Chills dco:SimpleAcutePyelonephritis) fl:sigma 0.\}.

$\{($ P dco:Fever $)$ fl:mu 0. $\}=>\{($ dco:FlankPain dco:SimpleAcutePyelonephritis $)$ fl:sigma 0.

(dco:Fever dco:SimpleAcutePyelonephritis) fl:sigma 0.

(dco:UrineDipstickTest dco:SimpleAcutePyelonephritis) fl:sigma 0.

(dco:PainfulKidneysPalpation dco:SimpleAcutePyelonephritis) fl:sigma 0

(dco:Chillsdco:SimpleAcutePyelonephritis) fl:sigma 0).

\section{References}

1. Pauker SG, Gorry GA, Kassirer JP, Schwartz WB (1976) Towards the simulation of clinical cognition. Taking a present illness by computer. Am J Med 65: 981-996.

2. Hersch WR, Lunin LF (1995) Perspectives on medical informatics: information technology in health care. Introduction and overview. J Am Soc Inf Sci 46:726728

3. Peleg M, Tu S (2006) Decision support, knowledge representation and management in medicine. Methods Inf Med. 45 1:72-80.

4. Sittig DF, Wright A, Osheroff JA, Middleton B, Teich JM, et al. (2008) Grand challenges in clinical decision support. J Biomed Inform 41: 387-392. 
Citation: Douali N, Papageorgiou El, Roo JD, Cools H, Jaulent MC (2015) Clinical Decision Support System based on Fuzzy Cognitive Maps. J Comput Sci Syst Biol 8: 112-120. doi:10.4172/jcsb.1000177

5. Balas EA, Weingarten S, Garb CT, Blumenthal D, Boren SA, et al. (2000) Improving preventive care by prompting physicians. Arch Intern Med 160:301308.

6. Roshanov PS, You JJ, Dhaliwal J, Koff D, Mackay JA, et al. (2011) Can computerized clinical decision support systems improve practitioners' diagnostic test ordering behavior? A decision-maker-researcher partnership systematic review. Roshanov et al. Implementation Science 6: 88.

7. Kawamoto K, Houlihan CA, Balas EA, Lobach DF (2005) Improving clinical practice using clinical decision support systems: a systematic review of trials to identify features critical to success. BMJ 330:1-8.

8. Garg AX, Adhikari NK, McDonald H, Rosas-Arellano MP, Devereaux PJ, et al. (2005) Effects of computerized clinical decision support systems on practitioner performance and patient outcomes: a systematic review. JAMA 293:12231238

9. Kucher N, Koo S, Quiroz R, Cooper JM, Paterno MD, et al. (2005) Electronic alerts to prevent venous thromboembolism among hospitalized patients. N Engl J Med 352: 969-977

10. Institute of Medicine. Crossing the Quality Chasm: A New Health System for the 21st Century (2001) National Academy Press.

11. Schreiber G, Akkermans $H$, (2000) Knowledge Engineering and Management: The CommonKADS Methodology. Anjewierden A, Hoog DR, Shadbolt, Nigel, Van-de VW, Wielinga B. Cambridge, MA: The MIT Press.

12. Shiffman R, Brandt C, Liaw $Y$, Corb G (1999) A design model for computerbased guideline implementation based on information management services. J Am Med Inform Assoc 6: 99-103.

13. Gersende G (2006) Analyse Informatique de Guides de Bonnes Pratiques Cliniques.

14. Lin L, Paul JHH, Olivia RLS (2006) A decision support system for lower back pain diagnosis: Uncertainty management and clinical evaluations. Decision Support Systems Volume 42: 1152-1169.

15. Hai TZ, Yeong KB, Hong GK (2008) An Ontology-Based Bayesian Network Approach for Representing Uncertainty in Clinical Practice Guidelines. LNAI 5327:161-173

16. Stylios CD, Georgopoulos VC, Malandraki GA, Chouliara S (2008) Fuzzy cognitive map architectures for medical decision support systems. Appl Soft Comput 8: 1243-1251

17. Janice AL, John RB (2010) Urinary tract infection. Primary Care: Clinics in Office Practice 37: 491-507.

18. Rahul M, Sudhir A, Saroj S, Sanjay C, Kusum H (2009) Urinary tract infections caused by Pseudomonas aeruginosa: A minireview. J Infection and Public Health 2: 101-111.

19. Urinary tract infection in women. Tomas L. Griebling, MD.

20. Foxman B, Brown P (2003) Epidemiology of urinary tract infections: transmission and risk factors, incidence, and costs. Infect Dis Clin North Am 49: 53-70.

21. Mehnert-Kay SA (2005) Diagnosis and Management of uncomplicated urinary tract infections. Am Fam Physician 72: 451-456.

22. Gupta K, Hooton TM, Wobbe CL, Stamm WE (1999) The prevalence of antimicrobial resistance among uropathogens causing acute uncomplicated cystitis in young women. Int J Antimicrob Agents 11:305-308.

23. Robertson J, Walkom E, Pearson SA, Hains I, Williamsone M, et al. (2010) The impact of pharmacy computerised clinical decision support on prescribing, clinical and patient outcomes: a systematic review of the literature. Int J Pharm Pract 18:69-87.

24. Berner ES (2007) Clinical Decision Support Systems: Theory and Practice, Springer.

25. Schurink CAM, Lucas PJF, Hoepelman IM, Bonten MJM (2005) Computerassisted decision support for the diagnosis and treatment of infectious diseases in intensive care units. Lancet Infect Dis 5: 305-312.

26. Kosko B (1986) Fuzzy Cognitive Maps. International Journal Man-Machine Studies $24: 65-75$
27. Douali N, Huszka C, Roo DJ, Papageorgiou El, Jaulent MC, et al. (2014) Diagnosis Support Systems based on clinical guidelines: a Comparison of Case-Based Fuzzy Cognitive Maps and Bayesian Networks. Comput Methods Programs Biomed Journal. 113: 133-143.

28. Douali N, Abdennour M, Zucker JD, Jaulent MC (2014) Formalization of Clinica Practice Guidelines: Nonalcoholic Steatohepatitis Diagnosis Model-Related Personalized Medicine. EJBI 10: 6-10.

29. Douali N, Abdennour M, Sasso M, Miette V, Tordjman J, et al. (2013) Noninvasive diagnosis of nonalcoholic steatohepatitis disease based on clinical decision support system. Stud Health Technol Inform 192:1178.

30. Douali N, Roo JD, Jaulent MC (2012) Decision support system based semantic web for personalized patient care. Stud Health Technol Inform 180:1203-1205.

31. Douali N, Roo JD, Jaulent MC (2012) Clinical diagnosis support system based on case based fuzzy cognitive maps and semantic web. Stud Health Technol Inform 180: 295-299.

32. Papageorgiou El, Huszka C, Roo JD, Douali N, Jaulent MC, et al. (2013) Application of probabilistic and fuzzy cognitive approaches in semantic web framework for medical decision support. Comput Methods Programs Biomed 112:580-598.

33. Douali N, Jaulent MC (2013) Integrate personalized medicine into clinical practice to improve patient safety. IRBM Journal 34: 53-55.

34. Douali N, Jaulent MC (2013) Clinical Practice Guidelines Formalization for Personalized Medicine. IJAEC 4: 26-33.

35. Douali N, De Roo J, Papageorgiou E I, Jaulent MC (2011) Case Based Fuzzy Cognitive Maps (CBFCM): New method for medical reasoning: Comparison study between CBFCM/FCM. FUZZ 20:844-850

36. Douali N, Jaulent MC (2012) Genomic and personalized medicine decision support system. In Procedings IEEE ICCS: 1-4.

37. Douali N, Papageorgiou El, Roo JD, Sun H, Colaert D, et al. (2013) Improve treatment of pneumonia and reduce adverse drug events. In Procedings IEEE EMBS Point-of-Care Healthcare Technologies : 89-92.

38. Douali N, Roo JD, Vanel JM, Jaulent MC (2013) New Semantic Web rules and new medical reasoning framework. In Procedings Instrumentation and Measurement Technology Conference (I2MTC): 1332-1336.

39. Papageorgiou EI, Spyridonos PP, Glotsos DT, Stylios CD, Ravazoula P, et al (2008) Brain tumor characterization using the soft computing technique of fuzzy cognitive maps. Applied Soft Computing 8: 820-828.

40. Georgopoulos VC, Malandraki GA, Stylios CD (2003). A fuzzy cognitive map approach to differential diagnosis of specific language impairment. Artificial intelligence in Medicine 29: 261-278.

41. Glykas G (2010) Fuzzy cognitive maps: theory, methodologies, tools and 1103 applications. Springer Verlag.

42. Douali N, Papageorgiou El, De Roo J, Jaulent MC (2011) Case Based Fuzzy Cognitive Maps (CBFCM): New method for medical reasoning: Comparison study between CBFCM/FCM. (FUZZ), 2011 IEEE International Conference on fuzzy sets, 844-850.

43. Schober D, Boeker M, Bullenkamp J, Huszka C, Depraetere K, et al. (2010)The DebugIT core ontology: semantic integration of antibiotic resistance patterns. Stud Health Technol Inform 160:1060-1064

44. Jos De Roo. Eye note.

45. Pauker SG, Gorry GA, Kassirer JP, Schwartz WB (1976) Towards the simulation of clinical cognition. Taking a present illness by computer. Am J Med 60: 981-996.

46. Sittig DF, Wright A, Osheroff JA, Middleton B, Teich JM, et al. (2008) Grand challenges in clinical decision support. J Biomed Inform 41: 387-392.

47. Balas EA, Weingarten S, Garb CT, Blumenthal D, Boren SA, et al. (2000) Improving preventive care by prompting physicians. Arch Intern Med 160: 301 308 
Citation: Douali N, Papageorgiou EI, Roo JD, Cools H, Jaulent MC (2015) Clinical Decision Support System based on Fuzzy Cognitive Maps. J Comput Sci Syst Biol 8: 112-120. doi:10.4172/jcsb.1000177

48. Kawamoto K, Houlihan CA, Balas EA, Lobach DF (2005) Improving clinical practice using clinical decision support systems: a systematic review of trials to identify features critical to success. BMJ 330: 765.

49. Garg AX, Adhikari NK, McDonald H, Rosas-Arellano MP, Devereaux PJ, et al.
(2005) Effects of computerized clinical decision support systems on practitioner performance and patient outcomes: a systematic review. JAMA 293: 12231238.

50. Kucher N, Koo S, Quiroz R, Cooper JM, Paterno MD, et al. (2005) Electronic alerts to prevent venous thromboembolism among hospitalized patients. J Med 352: 969-977. 